\title{
Utilisation des images d'observation de la terre pour améliorer la cartographie des risques de maladies associées au changement climatique
}

\author{
SO Kotchi ${ }^{1 *}$, C Bouchard ${ }^{1}$, A Ludwig', EE Rees ${ }^{1}$, S Brazeau ${ }^{1}$
}

\section{Résumé}

Le nombre de cas de plusieurs maladies infectieuses liées au climat chez l'humain, y compris les maladies transmises par les tiques et les moustiques, a augmenté au Canada et dans d'autres parties du monde depuis la fin du siècle dernier. Une nouvelle méthode utilisant des images satellites pour cerner les déterminants environnementaux et climatiques permettant de prévoir et de cartographier les risques associés à ces maladies contribue à appuyer les activités de recherche, de surveillance, de prévention et de contrôle, et aider à mieux évaluer les impacts du changement climatique au Canada. Il est possible d'utiliser les images d'observation de la Terre afin de surveiller les changements à la surface de la planète et dans l'atmosphère de façon systématique, et ce, à différentes échelles temporelles et spatiales. Ces images peuvent éclairer l'estimation et la surveillance des déterminants environnementaux et climatiques, et donc aussi la prévision des maladies et la cartographie des risques. Le réseau actuel de satellites d'observation de la Terre produit une grande quantité et une grande variété de données. Ces données présentent différentes caractéristiques en termes de précision et de résolution spatiales, temporelles et thématiques. Le présent aperçu vise à décrire comment les images d'observation de la Terre peuvent éclairer l'évaluation des risques et la cartographie des maladies transmises par les tiques et les moustiques au Canada, leurs potentiels avantages et limites, les répercussions et les prochaines étapes.

Citation suggérée : Kotchi SO, Bouchard C, Ludwig A, Rees EE, Brazeau S. Utilisation des images d'observation de la terre pour améliorer la cartographie des risques de maladies associées au changement climatiques. Relevé des maladies transmissibles au Canada, 2019;45(5):148-58. https://doi.org/10.14745/ccdr.v45i05a04f

Mots-clés : Images d'observation de la Terre, changement climatique, cartographie des risques, maladies infectieuses, maladies transmises par les tiques, maladies transmises par les moustiques, télédétection, déterminants environnementaux, déterminants climatiques, Canada

\section{Introduction}

Le changement climatique a entraîné une hausse des températures et du niveau des océans, une variabilité climatique accrue et des changements dans la fréquence et l'intensité des précipitations (1,2). Les premiers signes de changements climatiques au Canada remontent aux années 1950 (3-6) et la température moyenne a augmenté de plus de $1,5^{\circ} \mathrm{C}$ entre 1950 et 2010 , soit près du double de la moyenne mondiale (7). L'ampleur de ce changement varie d'une région à l'autre, les changements les plus importants s'étant produits dans le Nord du Canada et plus particulièrement dans l'Arctique (8). Les taux d'humidité moyens au Canada ont également augmenté, avec une augmentation moyenne des précipitations d'environ $12 \%$ (8). Ces tendances devraient se poursuivre, avec une augmentation de l'intensité et de la fréquence des phénomènes météorologiques extrêmes tels que les vagues de chaleur, les sécheresses, les inondations et les feux de forêt (5,6,8-10). L'urbanisation et la création d'îlots de chaleur qui en découle ont contribué à ces changements climatiques (11).

Le climat a un impact direct sur les mouvements des populations humaines et des vecteurs de maladies infectieuses, tels que les tiques et les moustiques, et sur leurs populations hôtes $(12,13)$. L'augmentation de la température et la variabilité du climat entraînent une augmentation et une expansion géographique des populations de vecteurs et des maladies qu'ils transmettent $(1,7,11,14-22)$. Par exemple, de nombreuses études ont démontré l'expansion des tiques vers le Nord, ce qui a été associé à une augmentation constante du nombre de cas de la maladie de Lyme chez l'humain (23-25). Une étude prévoit que l'incidence du virus du Nil occidental augmentera probablement de plus de 17 fois d'ici 2050 (26). Avec l'expansion vers le Nord du moustique Aedes albopictus aux États-Unis (É.-U.) $(27,28)$, I'Amérique du Nord pourrait être confrontée à des maladies exotiques transmises par les moustiques comme la dengue, le Zika, la fièvre jaune et le Chikungunya. D'autres maladies transmises par les tiques et les

Page $148 \quad$ RMTC • le 2 mai $2019 \bullet$ Volume 45-5 
moustiques sont susceptibles d'augmenter ou d'émerger au Canada en raison du réchauffement planétaire $(26,27,29-31)$.

En raison de l'interaction entre le changement climatique et les maladies à vecteurs, il est de plus en plus important de pouvoir mesurer les changements de température, de précipitations et autres variables relatives à l'habitat. Le Canada est un vaste pays et les stratégies de surveillance actuelles ne sont pas conçues pour surveiller l'impact du changement climatique sur la propagation géographique des maladies transmises par les tiques et les moustiques. Les outils d'observation de la Terre sont de plus en plus utilisés d'une façon qui accroît notre capacité à effectuer cette surveillance. L'observation de la Terre désigne l'acquisition, le traitement, l'analyse, l'interprétation et la diffusion d'informations physiques, chimiques et biologiques sur la terre, les océans et l'atmosphère au moyen de capteurs de télédétection satellitaire, aéroportée ou in situ. Les technologies de télédétection, qui mesurent les propriétés d'un objet au moyen d'ondes électromagnétiques, peuvent être utilisées en combinaison avec de nombreux autres capteurs terrestres comme les stations météorologiques et les ballons-sondes (32).

Cet aperçu porte sur les images d'observation de la Terre acquises par les satellites de télédétection. Les images d'observation de la Terre et leurs dérivés fournissent des données sur la température, les précipitations, I'humidité, la forêt, les terres humides, l'agriculture, les milieux bâtis et plus encore. Plusieurs études ont démontré l'efficacité des images d'observation de la Terre et de leurs dérivés pour l'évaluation des risques et la cartographie des maladies transmises par les tiques et les moustiques dans le monde. Le Malaria Atlas Project (MAP) $(33,34)$, I'Epidemic Prognosis Incorporating Disease and Environmental Monitoring for Integrated Assessment (EPIDEMIA) (35) et le Mapping Malaria Risk in Africa (MARA) $(36,37)$ sont des exemples de programmes de surveillance qui ont recours aux images d'observation de la Terre et leurs dérivés. Les images d'observation de la Terre sont également utilisées pour suivre les maladies transmises par les tiques et les moustiques et évaluer les conditions géographiques et climatiques de leur propagation au Canada. Le présent aperçu vise à décrire comment les images d'observation de la Terre peuvent éclairer l'évaluation et la cartographie des risques des maladies transmises par les tiques et les moustiques au Canada afin d'en assurer la surveillance et à cerner leurs avantages et leurs limites éventuelles, les répercussions et les prochaines étapes.

\section{Les images d'observation de la Terre pour l'évaluation et la cartographie des risques}

Les images d'observation de la Terre ne permettent évidemment pas d'observer directement les tiques et les moustiques dans leurs habitats. Elles permettent plutôt d'obtenir des données sur des variables et des indicateurs qui servent à caractériser les déterminants environnementaux et climatiques (DEC) qui influencent la présence et le développement des tiques et des moustiques. Les images d'observation de la Terre permettent d'obtenir les données sur les DEC grâce à plusieurs méthodes de traitement et d'analyse, y compris des corrections géométriques, radiométriques et atmosphériques, et des analyses de classification des images. Les DEC qui en résultent comprennent la température de surface, la température de l'air, I'humidité du sol, l'humidité de surface, la vapeur d'eau atmosphérique, l'humidité de l'air, la quantité de précipitations, la topographie, la couverture et l'épaisseur de la neige, le type de sol, le type de végétation et sa densité, les indices de végétation, les zones inondées, les milieux humides, les paramètres liés à la qualité des eaux (tels la concentration en chlorophylle, la matière organique dissoute, les sédiments en suspension, la couleur et la salinité), les forêts, les zones forestières, urbaines ou bâties ainsi que les zones agricoles $(33,38-40)$. Ainsi, de nombreux DEC dérivés des images d'observation de la Terre sont des déterminants importants de la survie et de l'abondance des vecteurs des maladies transmises par les tiques et les moustiques.

Le Tableau 1 présente une liste des satellites d'observation de la Terre les plus couramment utilisés, leurs capteurs et les DEC qui en sont dérivés.

\section{Maladies transmises par les moustiques}

Les déterminants environnementaux et climatiques (DEC) des maladies transmises par les moustiques comprennent à la fois les milieux anthropiques (comme les zones urbaines, périurbaines et rurales) et les milieux naturels (comme les forêts et les milieux humides) $(7,17,49-52)$. Ces DEC comportent également des facteurs climatiques et microclimatiques connus pour avoir une incidence sur la propagation des maladies transmises par les moustiques, comme la température, I'humidité et les précipitations (17,20,52-54). Certaines espèces de moustiques ont également des DEC particuliers. Des espèces, comme Aedes albopictus (qui propage les virus de la dengue, du Chikungunya et du Zika) ou Culex pipiens (qui propage le virus du Nil occidental), sont très bien adaptées aux milieux urbains. D'autres espèces, comme Culiseta melanura (qui propage l'encéphalite équine de l'Est), sont propres aux milieux humides naturels et se trouvent uniquement dans des zones rurales.

\section{Maladies transmises par les tiques}

Les DEC des maladies transmises par les tiques diffèrent également selon les espèces. Le vecteur de la maladie de Lyme, Ixodes scapularis, a évolué dans les biomes forestiers tempérés (feuillus mixtes) en Amérique du Nord, tandis que le vecteur du virus de Powassan, Ixodes cookei, vit principalement dans des terriers et plus rarement sur la végétation. Historiquement, les changements dans la répartition de divers types d'habitats et des espèces hôtes ont été accompagnés de changements dans la répartition et l'abondance des tiques (55). Les DEC des maladies transmises par les tiques comprennent le type et la densité du couvert forestier et le degré de fragmentation de la forêt, ainsi que les conditions de température et d'humidité prévalant dans les habitats forestiers $(12,13,17,56-63)$. 
Tableau 1 : Images d'observation de la Terre en service en accès libre et les déterminants environnementaux et climatiques qui en sont dérivés

\begin{tabular}{|c|c|c|c|c|c|c|c|c|c|c|c|c|c|c|}
\hline \multirow[b]{2}{*}{$\begin{array}{c}\text { Satellite } \\
\text { Nom }\end{array}$} & \multirow[b]{2}{*}{ Capteur } & \multirow[b]{2}{*}{$\begin{array}{c}\text { Rés. } \\
\text { spatiale }\end{array}$} & \multirow[b]{2}{*}{$\begin{array}{l}\text { Rés. } \\
\text { temp. }\end{array}$} & \multicolumn{11}{|c|}{ Déterminants environnementaux et climatiques dérivés } \\
\hline & & & & $\begin{array}{l}\text { Couv. } \\
\text { sols }\end{array}$ & $\begin{array}{c}\text { Type } \\
\text { sol }\end{array}$ & $\begin{array}{l}\text { Type } \\
\text { vég. }\end{array}$ & $\begin{array}{l}\text { Qté } \\
\text { vég. }\end{array}$ & $\begin{array}{l}\text { Prof. } \\
\text { couv. } \\
\text { neige }\end{array}$ & $\begin{array}{c}\text { Qual. } \\
\text { eau }\end{array}$ & $\begin{array}{l}\text { Hum. } \\
\text { surf. }\end{array}$ & $\begin{array}{c}\text { Temp. } \\
\text { surf. }\end{array}$ & $\begin{array}{l}\text { Temp. } \\
\text { air }\end{array}$ & $\begin{array}{l}\text { Vap. } \\
\text { d'eau }\end{array}$ & Préc. \\
\hline Aqua & AIRS & $2,3-41 \mathrm{~km}$ & 0,5 jour & - & - & - & - & - & - & - & Y & Y & - & - \\
\hline DMSP & $\mathrm{SSM} / \mathrm{I}$ & $13-69 \mathrm{~km}$ & 1 jour & - & - & - & - & $Y$ & - & - & - & - & $Y$ & $Y$ \\
\hline GCOM-W1 & AMSR-2 & $3-62 \mathrm{~km}$ & 1 jour & - & - & - & - & $Y$ & $Y$ & - & - & - & $Y$ & $Y$ \\
\hline GPM & DPR & $5 \mathrm{~km}$ & $\begin{array}{l}1-2 \\
\text { heures }\end{array}$ & - & - & - & - & - & - & - & - & - & - & $Y$ \\
\hline GPM & GMI & 4-32 km & $\begin{array}{l}1-2 \\
\text { heures }\end{array}$ & - & - & - & - & - & - & - & $Y$ & - & $Y$ & $Y$ \\
\hline Landsat-5 & TM & $30-120 \mathrm{~m}$ & 16 jours & $Y$ & $Y$ & $Y$ & $Y$ & $Y$ & - & $Y$ & $Y$ & - & - & - \\
\hline Landsat-7 & ETM+ & $15-60 \mathrm{~m}$ & 16 jours & $Y$ & $Y$ & $Y$ & $Y$ & $Y$ & - & $Y$ & Y & - & - & - \\
\hline Landsat-8 & OLI & $15-30 \mathrm{~m}$ & 16 jours & $Y$ & $Y$ & $Y$ & $Y$ & $Y$ & - & - & - & - & - & - \\
\hline Landsat-8 & TIRS & $100 \mathrm{~m}$ & 8 jours & - & - & - & - & - & - & - & $Y$ & - & $Y$ & - \\
\hline MSG & SEVIRI & $1-4,8 \mathrm{~km}$ & $15 \mathrm{~min}$ & - & - & - & $Y$ & - & - & $Y$ & Y & - & $Y$ & $Y$ \\
\hline $\begin{array}{l}\text { NOAA } \\
15-19\end{array}$ & AVHRR/3 & $1,1 \mathrm{~km}$ & 0,5 jour & $Y$ & - & - & $Y$ & $Y$ & - & $Y$ & $Y$ & - & $Y$ & - \\
\hline Sentinel-1 & C-SAR & $5-00 \mathrm{~m}$ & 12 jours & $Y$ & - & $Y$ & $Y$ & $Y$ & - & - & - & - & - & - \\
\hline Sentinel-2 & MSI & $10-60 \mathrm{~m}$ & 5 jours & $Y$ & $Y$ & $Y$ & $Y$ & $Y$ & - & - & - & - & - & - \\
\hline Sentinel-3 & $\mathrm{OLCl}$ & $300 \mathrm{~m}$ & 2 jours & $Y$ & - & - & $Y$ & - & $Y$ & - & - & - & - & - \\
\hline Sentinel-3 & SLSTR & $\begin{array}{l}500-1000 \\
m\end{array}$ & $\begin{array}{l}1-4 \\
\text { jours }\end{array}$ & - & - & - & $Y$ & Y & - & - & Y & - & - & - \\
\hline SMAP & MWR & $40 \mathrm{~km}$ & 1,5 jour & - & - & - & - & - & Y & Y & - & - & - & - \\
\hline SNPP & ATMS & $16-75 \mathrm{~km}$ & 0,5 jour & - & - & - & - & - & - & $Y$ & - & $Y$ & - & - \\
\hline SNPP & VIIRS & $\begin{array}{l}375-750 \\
\mathrm{~m}\end{array}$ & $6 \mathrm{~min}$ & - & - & - & $Y$ & $Y$ & - & $Y$ & $Y$ & - & $Y$ & - \\
\hline Terra & ASTER & $15-90 \mathrm{~m}$ & 5 jours & $Y$ & $Y$ & - & $Y$ & $Y$ & - & - & $Y$ & - & $Y$ & - \\
\hline $\begin{array}{l}\text { Terra, } \\
\text { Aqua }\end{array}$ & MODIS & $\begin{array}{l}250-1000 \\
\mathrm{~m}\end{array}$ & $5 \mathrm{~min}$ & $Y$ & $Y$ & $Y$ & $Y$ & $Y$ & $Y$ & $Y$ & $\mathrm{Y}$ & $Y$ & $Y$ & - \\
\hline
\end{tabular}

Abréviations : Couv., couverture; Hum., Humidité; Préc., précipitations; Qté, quantité; Qual., qualité; Rés., résolution, Surf. Surface; Temp., température; Vap., Vapeur; Vég., végétation; Y, images acquises par le système satellite/capteur; -, sans objet

Noms des satellites: DMSP, Defense Meteorological Satellite Program; GCOM, Global Change Observation Mission; GPM, Global Precipitation Measurement Mission; MSG, Meteosat Second Generation; NOAA, National Oceanic and Atmospheric Administration; SMAP, Soil Moisture Active Passive; SNPP, Suomi National Polar-orbiting Partnership Satellite

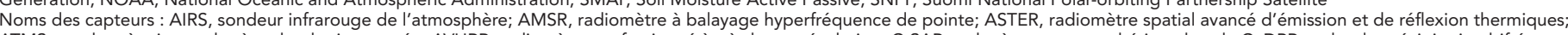

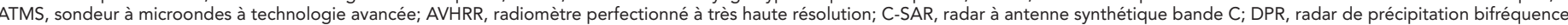

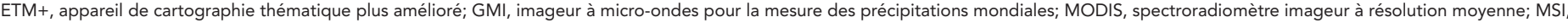

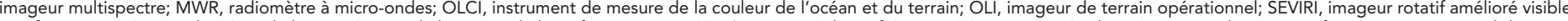

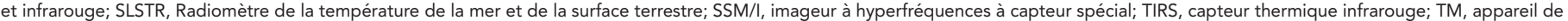
cartographie thématique; VIIRS, radiomètre à balayage dans le visible et l'infrarouge (41-48)

\section{Évaluations des risques}

Il est possible d'utiliser les DEC dérivés des images d'observation de la Terre pour évaluer les risques associés à différentes maladies liées au climat $(49,50,52,64-69)$. La possibilité d'une infection chez l'humain dépend d'une série de risques: le risque que les vecteurs en cause soient présents dans l'environnement; le risque que ces vecteurs soient contaminés par un pathogène; le risque que les populations humaines soient exposées à ces vecteurs; le risque de transmission de la maladie. Les DEC, dérivés des images d'observation de la Terre, combinés aux données de surveillance, permettent d'évaluer, de modéliser et de cartographier ces différentes composantes du risque. Les images d'observation de la Terre et leurs dérivés peuvent servir à cartographier les risques sur une base régulière une fois que le modèle de risque basé sur ces données est développé et validé.

L'utilisation la plus courante des images d'observation de la Terre pour l'évaluation et la cartographie des risques est la surveillance des risques environnementaux associés à la présence ou à l'abondance des tiques et des moustiques. Les DEC dérivés des images d'observation de la Terre peuvent également être combinés avec des données de cas humain pour évaluer et cartographier directement le risque de transmission de la maladie. La figure 1 présente un exemple de structure 
Figure 1 : Structure opérationnelle fondée sur l'observation de la Terre pour l'évaluation et la cartographie des risques de maladies infectieuses liées au climat

\section{Acquisition, traitement et intégration des données d'observation de la Terre}

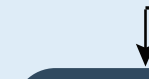

Données

météorologiques

Modélisation des risques et validation

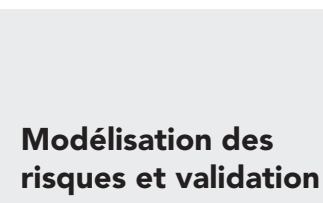

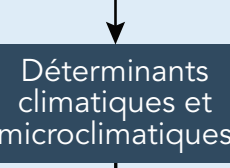

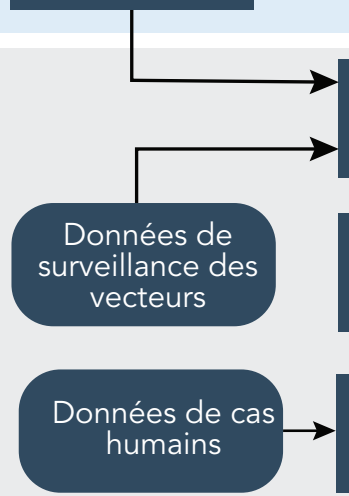

Images d'observation de la Terre (IOT)

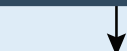

Estimation des déterminants environnementaux et climatiques

Traitement et

intégration

des images

multicapteurs

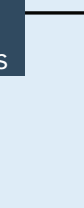

Cartographie des risques

Carte de risque de présence/abondance des vecteurs
Carte de risque d'exposition à des vecteurs infectés
Carte de risque de transmission de la maladie
Résultats pour les interventions en santé publique
Estimation de la population à risque
Zones cibles pour la surveillance active des vecteurs
Zones cibles de

contrôle et de

prévention opérationnelle fondée sur l'observation de la Terre que l'on peut utiliser pour l'évaluation et la cartographie des risques de maladies infectieuses liées au climat.

\section{Avantages et limites}

Les données provenant des images d'observation de la Terre et de leurs dérivés présentent de nombreux avantages par rapport aux autres types de données pour estimer les DEC et cartographier le risque de transmission des maladies infectieuses liées au climat $(17,22,70,71)$. Les images d'observation de la Terre présentent au moins cinq avantages potentiels pour l'évaluation des risques pour la santé publique liés aux maladies à vecteurs au Canada.

Premièrement, le Canada est un vaste pays qui n'est pas actuellement entièrement couvert par des données d'observation sur le terrain, utilisées dans les méthodes traditionnelles de surveillance (p.ex. piégeage et analyse de la densité et du type de moustiques ou cartographie des habitats des tiques par la recherche des tiques). Ces données de surveillance traditionnelles ne couvrent généralement qu'un nombre limité de sites de surveillance dont la répartition est inégale entre les provinces. Les images d'observation de la Terre couvrent quant à elles des surfaces continues du pays, où chaque point du territoire observé est un " site de surveillance ". De plus, ces images peuvent couvrir de vastes régions géographiques comme le Canada dans un délai (minutes, heures ou jours, selon le satellite utilisé) qui ne peut être égalé par les méthodes de surveillance traditionnelles.

Deuxièmement, le Canada compte de nombreuses régions éloignées difficiles d'accès. Ces régions sont couvertes par des images d'observation de la Terre au même titre que le reste du pays. Ceci offre l'avantage unique de pouvoir surveiller de 
façon continue même les régions les plus éloignées. Cela est particulièrement important au Canada, car certaines de ces régions éloignées, comme l'Arctique, sont affectées de façon disproportionnée par le changement climatique.

Troisièmement, l'utilisation d'images d'observation de la Terre permet de répondre au problème du coût élevé de la surveillance active sur une vaste zone géographique. II est possible d'accéder librement aux images d'observation de la Terre acquises par plusieurs satellites sur des plateformes de données ouvertes ou à faible coût. Au Canada, il existe une grande quantité de données dérivées d'images d'observation de la Terre prêtes à l'emploi. Ces données sont produites par des ministères fédéraux comme Agriculture et Agroalimentaire Canada, Ressources naturelles Canada et Environnement et Changement climatique Canada. Elles sont disponibles gratuitement sur le site Web ouvert du gouvernement (https:// ouvert.canada.ca/fr/donnees-ouvertes).
Quatrièmement, les méthodes et protocoles de surveillance active varient selon les projets, les années, les programmes et les administrations. En raison de cette variabilité, il est difficile d'établir des comparaisons dans le temps et entre divers lieux. Pour leur part, l'acquisition des images d'observation de la Terre se fait de façon standardisée et sur une base régulière. Cela permet de comparer les détections de signaux (p.ex. situations anormales et alertes) d'une manière plus efficace dans l'ensemble du pays.

Cinquièmement, les satellites d'observation de la Terre peuvent être mobilisés rapidement pour appuyer des opérations d'urgence en temps réel en cas de catastrophe majeure qui présente des risques pour la santé publique. Par exemple, dans le cas d'une inondation, il est possible d'utiliser des images d'observation de la Terre pour délimiter et surveiller les zones à risque où des activités de lutte contre les moustiques peuvent être requises. La Charte internationale « Espace et catastrophes majeures » (72) a pour but de faciliter la mobilisation des satellites d'observation de la Terre de diverses agences

\section{Tableau 2 : Avantages de l'utilisation des images d'observation de la Terre pour l'estimation des déterminants environnementaux et climatiques et la cartographie des risques de maladies infectieuses liées au climat \\ Avantage \\ Description}

Mesures précises et régulières à différentes résolutions spatiales et temporelles
L'homogénéité et la régularité des observations faites à l'aide d'images d'observation de la Terre permettent de mesurer avec plus de précision les changements survenus au fil du temps, comme les changements environnementaux et climatiques, comparativement aux données recueillies sur le terrain. Ces mesures sont effectuées à différentes résolutions spatiales et temporelles, ce qui permet d'observer des phénomènes (par exemple, habitats des vecteurs, conditions microclimatiques) et leurs changements selon un spectre varié d'échelles allant de 0,31 m à $75 \mathrm{~km}$ et de fréquences temporelles allant de 5 minutes à 16 jours. Cette capacité d'observation multiéchelle qu'offrent les images d'observation de la Terre est unique et permet d'estimer les DEC et de cartographier les risques à l'échelle locale, régionale et globale en fonction des objectifs de santé publique.

Haute densité spatiale des observations, avec la couverture de vastes territoires, de régions éloignées et de zones difficiles d'accès

Les mesures effectuées à l'aide d'images d'observation de la Terre consistent en des données continues couvrant l'ensemble de la zone géographique couverte par le capteur. Il est possible d'estimer les DEC et de cerner les zones à faible risque ou à risque élevé pour toutes les parties du territoire. Les images d'observation de la Terre couvrent d'immenses territoires qui ne peuvent être échantillonnés avec des données recueillies sur le terrain. La plupart des satellites d'observation de la Terre peuvent capter des images couvrant la planète entière, y compris les régions éloignées et les zones géographiques difficiles d'accès. Ceci est très utile pour appuyer les initiatives de santé publique qui ciblent les collectivités éloignées.

Récurrence des observations sur une longue période

Les images d'observation de la Terre sont acquises de façon récurrente depuis près de 40 ans. La capacité d'observation d'un territoire donné sur de longues périodes offre une excellente occasion d'étudier et de prévoir l'impact du changement climatique sur l'émergence et la réémergence des maladies liées au climat.

Dérivés d'images d'observation de la Terre prêts à l'emploi

De nombreux produits prêts à l'emploi ont été développés à partir d'images d'observation de la Terre pour rendre ces données accessibles à une communauté plus large de personnes non expertes dans le traitement et l'analyse de ces images. Ces produits se rapportent à la fois aux déterminants environnementaux et aux déterminants climatiques.

Accès à de grandes quantités de données à faible coût

Plus de 1700 satellites d'observation de la Terre sont en service (74). Les images d'observation de la Terre de ces nombreux satellites ainsi que la plupart des dérivés sont accessibles gratuitement sur des plateformes de données ouvertes. La majorité des images commerciales d'observation de la Terre comportent des données à très haute résolution spatiale (moins de $2 \mathrm{~m}$ ). Les coûts associés à ces données diminuent rapidement avec l'augmentation du nombre de systèmes d'observation de la Terre et l'amélioration de leur performance.

Rapidité de mobilisation de nombreux satellites pour appuyer les opérations d'urgence

En vertu de la Charte internationale "Espace et catastrophes majeures » (72), les satellites et les services peuvent être rapidement mobilisés par de nombreuses agences spatiales pour appuyer la gestion des situations d'urgence dans les grandes zones sinistrées. Ils permettent d'évaluer rapidement les risques d'épidémies dans ces zones alors que le nombre de catastrophes majeures augmente avec le changement climatique. La Charte est en vigueur depuis I'an 2000. Elle a été mobilisée pour appuyer les interventions liées à l'éclosion du virus Ebola en Afrique de l'Ouest en 2014 (73). C'était la première fois que la Charte était mobilisée pour la gestion d'une maladie infectieuse. 
spatiales à l'appui des opérations d'urgence liées aux grandes catastrophes. Cette charte a été appliquée pour la première fois dans le cadre d'une maladie infectieuse lors de l'épidémie d'Ebola en Afrique de l'Ouest en 2014 (73). Le tableau 2 résume les avantages de l'utilisation des images d'observation de la Terre et de leurs dérivés.

\section{Limites}

L'utilisation des images d'observation de la Terre et de leurs dérivés comporte un certain nombre de limites. Premièrement, les dérivés d'images d'observation de la Terre sont conçus à I'origine pour d'autres domaines d'application, notamment l'agriculture, la foresterie et la conservation de la nature, et les indicateurs de qualité des données relatifs à ces dérivés sont principalement destinés à répondre aux objectifs de ces applications initiales. Cela signifie qu'il y a un manque de dérivés d'images d'observation de la Terre spécifiquement développés pour des maladies infectieuses comme les maladies transmises par les tiques et les moustiques. Par conséquent, plusieurs indicateurs de qualité des données (couverture géographique, résolution spatiale, résolution temporelle, échelle d'agrégation temporelle, fréquence de mise à jour, période d'archivage, composition des classes thématiques et exactitude des données) relatifs aux images d'observation de la Terre communément utilisées et leurs dérivés ne répondent pas aux critères de qualité des données nécessaires à l'estimation des DEC associés aux maladies infectieuses liées au climat.

Deuxièmement, les images d'observation de la Terre les plus utilisées pour l'estimation des déterminants climatiques et microclimatiques ont rarement une résolution spatiale et temporelle élevée. Par exemple, la température de surface et I'humidité du sol peuvent varier considérablement d'un endroit à l'autre et dans le temps. Les images d'observation de la Terre utilisées pour les calculer ne permettent pas de combiner une résolution spatiale élevée (moins de $30 \mathrm{~m}$ ) avec une résolution temporelle élevée (moins d'un jour) $(17,68,75)$; cependant, cette combinaison est nécessaire pour caractériser la dynamique des conditions microclimatiques et les microhabitats des vecteurs. Ainsi, ces données ne permettent pas toujours de mener des activités de contrôle et de prévention à l'échelle locale (p. ex. pour une municipalité).

Troisièmement, I'utilisation des images d'observation de la Terre et de leurs dérivés pour estimer les DEC à une échelle fine et pour cartographier les risques sur de longues périodes et sur de vastes zones géographiques génère une quantité considérable de données. Les méthodes et technologies classiques utilisées pour le traitement, l'analyse, le stockage et la gestion de données aussi volumineuses sont actuellement limitées.

Le tableau 3 résume ces limites et d'autres limitations associées à l'utilisation des images d'observation de la Terre et de leurs dérivés.

\section{Discussion}

Le changement climatique favorise l'émergence et la réémergence des maladies transmises par les tiques et les moustiques au Canada. L'utilisation de DEC dérivés des images d'observation de la Terre permet de cartographier l'expansion géographique de ces vecteurs et d'évaluer les risques de maladies qu'ils transmettent. À l'heure actuelle, les images d'observation de la Terre peuvent couvrir toutes les régions urbaines, rurales et éloignées du Canada de façon standardisée et sur une base régulière, à des résolutions spatiales et temporelles variées. Cependant, la précision des dérivés d'images d'observation de la Terre est souvent inconnue.

La précision d'une cartographie du risque dépend largement de la qualité des données utilisées pour modéliser le risque et produire la carte de risque. Nous travaillons actuellement à améliorer la qualité des dérivés des images d'observation de la Terre. Par exemple, des images d'observation de la Terre ayant différentes caractéristiques (y compris la résolution spatiale et les bandes spectrales) peuvent être combinées par fusion d'images ou par réduction d'échelle spatiale pour produire des dérivés d'images d'observation de la Terre à valeur ajoutée qui peuvent répondre aux critères de qualité requis pour estimer les DEC. Ainsi, si on combine des images de température de surface des terres (TST) du capteur thermique infrarouge de Landsat-8 (100 m, 16 jours) avec des images TST du spectroradiomètre imageur à résolution moyenne (MODIS) (1000 m, 5 minutes), on peut obtenir une moyenne sur 8 jours des images LST avec une résolution spatiale de $100 \mathrm{~m}$ (78). Grâce à des combinaisons de données multicapteurs ou multiproduits, les possibilités d'estimation des DEC et d'évaluation et de cartographie des risques de maladies infectieuses liées au climat dans un contexte de changement environnemental rapide et de variabilité climatique accrue sont pratiquement infinies. De plus, le traitement et l'analyse des données massives dérivées de la combinaison de données provenant de plusieurs satellites nécessitent l'application de méthodes d'intelligence artificielle. Ce faisant, il serait possible de créer une structure opérationnelle éclairée par l'observation de la Terre pour l'évaluation et la cartographie rapides des risques (EO-OFRAM : Earth Observation-Informed Operational Framework for Rapid Risk Assessment and Mapping) des maladies infectieuses liées au climat. Une telle plateforme serait entièrement automatisée et dotée d'une interface utilisateur facile à utiliser qui intégrerait des données de surveillance et d'autres données contextuelles. Elle pourrait servir à répondre à des questions, visualiser les réponses sur des cartes et produire des rapports de situation afin d'éclairer la prise de décision sur les interventions en santé publique.

Les auteurs et leurs partenaires universitaires et gouvernementaux travaillent actuellement à l'élaboration d'indicateurs microclimatiques améliorés, d'indicateurs de microhabitat des vecteurs et d'un système d'intégration et 


\section{Tableau 3 : Limites des images d'observation de la Terre et de leurs dérivés}

\section{Description}

\section{Limite}

Résolution spatiale grossière

Faible résolution

temporelle
La résolution spatiale correspond à la taille du plus petit détail que l'on peut observer sur l'image. Une résolution spatiale inférieure à $30 \mathrm{~m}$ est généralement recommandée pour les applications cartographiques à une échelle locale. Les images d'observation de la Terre et leurs dérivés se rapportant aux déterminants climatiques et microclimatiques ont généralement une résolution spatiale grossière (plus de $1000 \mathrm{~m}$ ).

La résolution temporelle correspond à la fréquence temporelle à laquelle un satellite acquiert des images d'observation de la Terre pour une même région. Une résolution temporelle bimensuelle (16 jours) convient aux images d'observation de la Terre utilisées pour estimer les déterminants environnementaux de la couverture terrestre. Pour estimer les déterminants microclimatiques, une résolution temporelle pouvant servir à établir des moyennes quotidiennes est plus indiquée. Cependant, les images d'observation de la Terre en accès libre à haute résolution spatiale (p. ex. les images Landsat-8), couramment utilisées, n'ont pas cette résolution temporelle.

Précision inconnue ou faible

Les projections relatives au changement climatique indiquent des augmentations de température de $1{ }^{\circ} \mathrm{C}$ à plus de $5^{\circ} \mathrm{C}$ dans les latitudes élevées, à partir des années $1950(76,77)$. Une incertitude inférieure à $1{ }^{\circ} \mathrm{C}$ serait appropriée pour les images d'observation de la Terre et leurs dérivés liés à la température. Les métadonnées de nombreux dérivés d'images d'observation de la Terre n'indiquent pas leur précision, alors que cette précision peut être très variable et n'est en général pas disponible.

Composition des classes de couverture du sol incomplètes

Un dérivé d'image d'observation de la Terre relatif aux déterminants environnementaux doit contenir toutes les classes de couverture du sol qui constituent des déterminants environnementaux d'intérêt. Cependant, la composition des classes de couverture du sol d'un dérivé d'image d'observation de la Terre dépend de son producteur et de l'objectif de sa création. De plus, il n'existe pas de dérivé d'image d'observation de la Terre spécialement conçu pour estimer tous les DEC de chaque maladie infectieuse liée au climat.

Échelle d'agrégation temporelle inadéquate

L'échelle d'agrégation temporelle est le pas temporel utilisé pour agréger les données multitemporelles. Les échelles d'agrégation le plus souvent utilisées pour les dérivés des images d'observation de la Terre sont des moyennes quotidiennes, hebdomadaires, mensuelles et annuelles. L'échelle d'agrégation temporelle adéquate dépend des vecteurs de la maladie. Le cycle de vie des vecteurs de maladies transmises par les moustiques est très court (de quelques jours à quelques mois) si on le compare à celui des vecteurs de maladies transmises par les tiques (plusieurs années). Une échelle hebdomadaire est plus adéquate dans le premier cas et une échelle annuelle dans le second. Toutefois, pour certains DEC, il n'existe pas de dérivés d'images d'observation de la Terre conçus avec les échelles d'agrégation temporelle recherchées (par exemple, les accumulations annuelles des degrés-jours de surface, dérivées des images des températures de surface).

Mises à jour très espacées

Une fréquence de mise à jour annuelle est généralement adéquate pour les dérivés d'images d'observation de la Terre utilisés pour estimer les déterminants environnementaux. Toutefois, plusieurs de ces produits ne sont pas mis à jour annuellement.

Courte période d'archivage

Une période d'archivage relativement longue (plus de 15 ou 30 ans) peut être nécessaire pour étudier l'évolution des risques de maladies infectieuses dans le contexte du changement climatique. Cependant, la période d'archivage de plusieurs images d'observation de la Terre et de leurs dérivés n'est pas assez longue pour étudier l'impact du changement climatique sur les maladies infectieuses.

\section{Couverture} géographique incomplète

L'évaluation et la cartographie des risques de maladies infectieuses aux frontières nécessitent souvent des données couvrant plusieurs régions administratives différentes (p. ex. Québec/Ontario; Canada/États-Unis).

Méthodes et outils traditionnels non adaptés aux données massives des images d'observation de la Terre

Un ensemble de données d'images d'observation de la Terre couvrant un vaste pays comme le Canada, avec une résolution spatiale élevée, une résolution temporelle élevée, des mises à jour fréquentes et une longue période d'archivage, produira des données massives pour lesquelles les méthodes et outils traditionnels de traitement et de gestion des données d'observation de la Terre ne sont pas appropriés.

Abréviations : DEC, déterminants environnementaux et climatiques

de fusion des données. Ces outils novateurs amélioreront la précision de l'estimation des DEC et appuieront l'évaluation et la cartographie dynamiques et multi-échelles des risques des maladies infectieuses liées au climat par le biais de l'EO-OFRAM. Dans le cadre du Programme Solutions innovatrices Canada, l'Agence de la santé publique du Canada a lancé un défi à I'industrie concernant le traitement et l'analyse des données massives (79). Un meilleur traitement et une meilleure analyse des données massives, notamment par l'application de techniques novatrices d'apprentissage automatique (un sous-ensemble de l'intelligence artificielle), permettront de mieux exploiter le grand volume de données que produisent les images d'observation de la Terre. L'utilisation d'un EO-OFRAM basé sur l'intelligence artificielle en santé publique dépendra de son efficacité à évaluer les risques dans différents environnements et à différents niveaux de décision (local, provincial/territorial et national). La création d'un EO-OFRAM vise à équiper et optimiser les activités de suivi, de contrôle et de prévention à ces trois niveaux de gouvernance. Les principaux défis d'une telle initiative sont le financement, la participation des intervenants en santé publique des différents paliers de gouvernement et l'élaboration de normes communes.

\section{Conclusion}

Les risques de maladies infectieuses émergentes liées au climat varient considérablement d'une région géographique à l'autre et dans le temps. Les images d'observation de la Terre et leurs dérivés offrent de nombreux avantages pour caractériser cette hétérogénéité par l'estimation des déterminants environnementaux et climatiques, et la cartographie des risques associés à ces maladies. Grâce à l'élaboration et à l'application d'approches améliorées pour traiter et analyser les données volumineuses dérivées des images d'observation de la Terre, notamment l'apprentissage automatique, ainsi que 
I'élaboration d'indicateurs pour les microhabitats des vecteurs et les microclimats, ces images offrent certains outils novateurs pour améliorer notre capacité à modéliser et à cartographier le risque, et ainsi appuyer encore mieux les interventions en santé publique contre les maladies à vecteur.

\section{Déclaration des auteurs}

SOK - Conceptualisation, enquête, rédaction de l'ébauche originale, révision et modifications

$\mathrm{CB}$ - Rédaction de l'ébauche originale, révision et modifications

$A L$ - Rédaction de l'ébauche originale, révision et modifications

ER - Rédaction de l'ébauche originale, révision et modifications

$\mathrm{SB}$ - Rédaction de l'ébauche originale, révision et modifications

\section{Références}

1. Luber G, Prudent N. Climate change and human health. Trans Am Clin Climatol Assoc 2009;120:113-7. PubMed

2. Prudent N, Houghton A, Luber G. Assessing climate change and health vulnerability at the local level: travis County, Texas. Disasters 2016 Oct;40(4):740-52. DOI PubMed

3. Warren FJ, Lemmen DS, editors. Vivre avec les changements climatiques au Canada : perspectives des secteurs relatives aux impacts et à l'adaptation. Ottawa (ON) : Gouvernement du Canada; 2014. 286 p. www.rncan.gc.ca/sites/www.nrcan. gc.ca/files/earthsciences/pdf/assess/2014/pdf/Rapportcomplet_Fra.pdf

4. Du X, Shrestha NK, Wang J. Assessing climate change impacts on stream temperature in the Athabasca River Basin using SWAT equilibrium temperature model and its potential impacts on stream ecosystem. Sci Total Environ 2019 Feb;650(Pt 2):1872-81. DOI PubMed

5. Statistique Canada. L'activité humaine et l'environnement, 2007 et 2008. Section 1 : Changements climatiques au Canada. Ottawa (ON) : StatCan. (Accédé 2019-0225). https://www150.statcan.gc.ca/n1/pub/16-201x/2007000/10542-fra.htm

6. Lemmen DS, Warren FJ, Lacroix J, Bush E, editors. Vivre avec les changements climatiques au Canada 2008. Ottawa (ON): Gouvernement du Canada. 448 p. (Accédé 2019-02 25). https://www.rncan.gc.ca/environnement/ ressources/publications/impacts-adaptation/rapports/ evaluations/2008/10254

7. Ogden NH. Maladies à transmission vectorielle, changement climatique et conception urbaine. Relevé des maladies transmissibles au Canada 2016;42:225-6. DOI

8. Zhang L, Zhao Y, Hein-Griggs D, Barr L, Ciborowski JJ. Projected extreme temperature and precipitation of the Laurentian Great Lakes Basin. Global Planet Change 2019;172:325-35. DOI

9. Zhang L, Zhao Y, Hein-Griggs D, Ciborowski JJ. Projected monthly temperature changes of the Great Lakes Basin. Environ Res 2018 Nov; 167:453-67. DOI PubMed

\section{Conflit d'intérêts}

Aucun.

\section{Financement}

Ce travail a été réalisé grâce au soutien de l'Agence de la santé publique du Canada et de l'Agence spatiale canadienne.

10. Waits A, Emelyanova A, Oksanen A, Abass K, Rautio A. Human infectious diseases and the changing climate in the Arctic. Environ Int 2018 Dec;121(Pt 1):703-13. DOI PubMed

11. Mathieu K, Karmali M. Maladies à transmission vectorielle, changements climatiques et modes de vie sains en milieu urbain : prochaines étapes. Relevé des maladies transmissibles au Canada 2016;42:243-5. DOI

12. Simon JA, Marrotte RR, Desrosiers N, Fiset J, Gaitan J, Gonzalez A, Koffi JK, Lapointe FJ, Leighton PA, Lindsay LR, Logan T, Milord F, Ogden NH, Rogic A, Roy-Dufresne E, Suter D, Tessier N, Millien V. Climate change and habitat fragmentation drive the occurrence of Borrelia burgdorferi, the agent of Lyme disease, at the northeastern limit of its distribution. Evol Appl 2014 Aug;7(7):750-64. DOI PubMed

13. Brownstein JS, Skelly DK, Holford TR, Fish D. Forest fragmentation predicts local scale heterogeneity of Lyme disease risk. Oecologia 2005 Dec;146(3):469-75. DOI PubMed

14. Ogden NH, Bigras-Poulin M, O'Callaghan CJ, Barker IK, Lindsay LR, Maarouf A, Smoyer-Tomic KE, Waltner-Toews $D$, Charron D. A dynamic population model to investigate effects of climate on geographic range and seasonality of the tick Ixodes scapularis. Int J Parasitol 2005 Apr;35(4):375-89. DOI PubMed

15. Clow KM, Leighton PA, Ogden NH, Lindsay LR, Michel P, Pearl DL, Jardine CM. Northward range expansion of Ixodes scapularis evident over a short timescale in Ontario, Canada. PLoS One 2017 Dec;12(12):e0189393. DOI PubMed

16. Bouchard C, Beauchamp G, Nguon S, Trudel L, Milord F, Lindsay LR, Bélanger D, Ogden NH. Associations between Ixodes scapularis ticks and small mammal hosts in a newly endemic zone in southeastern Canada: implications for Borrelia burgdorferi transmission. Ticks Tick Borne Dis 2011 Dec;2(4):183-90. DOI PubMed

17. Kotchi SO, Brazeau S, Ludwig A, Aube G, Berthiaume P. Earth Observation and Indicators Pertaining to Determinants of Health - An Approach to Support Local Scale Characterization of Environmental Determinants of Vector-Borne Diseases. In: Ouwehand L, editor. Proceedings of Living Planet Symposium 2016; 2016 May 9-13; Prague, 
Czech Republic. Noordwijk (The Netherlands): ESA Communications; 2016. http://lps16.esa.int/

18. Karmali M, Weinstock D. Présentation de compte-rendu d'ateliers sur des maladies à transmission vectorielle émergentes et les milieux urbains. Relevé des maladies transmissibles au Canada 2016;42:220. DOI

19. Kulkarni MA. Propagation et répercussions des maladies à transmission vectorielle émergentes à l'échelle mondiale. Relevé des maladies transmissibles au Canada 2016;42:2212. DOI PubMed

20. Berrang-Ford L, Harper SL, Eckhardt R. Maladies à transmission vectorielle : mettre fin au débat entre déterminants climatiques et déterminants sociaux. Relevé des maladies transmissibles au Canada 2016;42:235-6. DOI

21. Savić S, Vidić B, Grgić Z, Potkonjak A, Spasojevic L. Emerging Vector-Borne Diseases - incidence through vectors. Front Public Health 2014 Dec;2:267. DOI PubMed

22. Brazeau S, Kotchi SO, Ludwing A, Turgeon P, Petcat Y, Aube G, Ogden NH.Tele-Epidemiology and Public Health in the Canadian Context. In: Ouwehand L, editor. Proceedings of Living Planet Symposium 2016; 2016 May 9-13; Prague, Czech Republic. Noordwijk (The Netherlands): ESA Communications; 2016. http://lps16.esa.int/

23. Gouvernement du Canada. 2019. Surveillance de la maladie de Lyme. Ottawa (ON) : ASPC. (Accédé 2019-02-26) https:// www.canada.ca/fr/sante-publique/services/maladies/ maladie-lyme/surveillance-maladie-lyme.html

24. Ogden NH, Koffi JK, Lindsay LR, Fleming S, Mombourquette DC, Sanford C, Badcock J, Gad RR, Jain-Sheehan N, Moore S, Russell C, Hobbs L, Baydack R, Graham-Derham S, Lachance L, Simmonds K, Scott AN. Surveillance de la maladie de Lyme au Canada, de 2009 à 2012. Relevé des maladies transmissibles au Canada 2015;41(6):152-68. DOI

25. Gasmi S, Ogden NH, Lindsay LR, Burns S, Fleming S, Badcock J, Hanan S, Gaulin C, Leblanc MA, Russell C, Nelder M, Hobbs L, Graham-Derham S, Lachance L, Scott AN, Galanis E, Koffi JK. Surveillance de la maladie de Lyme au Canada, de 2009 à 2015. Relevé des maladies transmissibles au Canada 2017;43(10):219-25. DOI

26. Chen CC, Jenkins E, Epp T, Waldner C, Curry PS, Soos C Climate change and West Nile virus in a highly endemic region of North America. Int J Environ Res Public Health 2013 Jul;10(7):3052-71. DOI PubMed

27. Ludwig A, Zheng H, Vrbova L, Drebot MA, Iranpour $M$, Lindsay LR. Augmentation du risque de maladies endémiques transmises par des moustiques au Canada en raison du changement climatique. Relevé des maladies transmissibles au Canada 2019;45(4):99-107. DOI

28. Armstrong PM, Andreadis TG, Shepard JJ, Thomas MC. Northern range expansion of the Asian tiger mosquito (Aedes albopictus): analysis of mosquito data from Connecticut, USA. PLoS Negl Trop Dis 2017 May;11(5):e0005623 eCollection 2017. DOl PubMed

29. Bouchard C, Dibernardo A, Koffi J, Wood H, Leighton PA, Lindsay LR. Augmentation du risque de maladies transmises par des tiques en raison des changements climatiques et environnementaux. Relevé des maladies transmissibles au Canada 2019;45(4):89-98. DOI

30. Sonenshine DE. Range Expansion of Tick Disease Vectors in North America: Implications for Spread of Tick-Borne Disease. Int J Environ Res Public Health 2018 Mar;15(3):E478. DOI PubMed

31. Hongoh V, Berrang-Ford L, Scott ME, Lindsay LR. Expanding geographical distribution of the mosquito, Culex pipiens, in Canada under climate change. Appl Geogr 2012;33(1):5362. DOI

32. Group on Earth Observations (GEO). What is Earth observation? (Accessed February 2019). https://www. earthobservations.org/g_faq.html

33. Clements AC, Reid HL, Kelly GC, Hay SI. Further shrinking the malaria map: how can geospatial science help to achieve malaria elimination? Lancet Infect Dis 2013 Aug;13(8):70918. DOl PubMed

34. Hay SI, Snow RW. The malaria Atlas Project: developing global maps of malaria risk. PLoS Med 2006 Dec;3(12):e473. DOI PubMed

35. Merkord CL, Liu Y, Mihretie A, Gebrehiwot T, Awoke W, Bayabil E, Henebry GM, Kassa GT, Lake M, Wimberly MC. Integrating malaria surveillance with climate data for outbreak detection and forecasting: the EPIDEMIA system. Malar J 2017 Feb;16(1):89. DOI PubMed

36. Thomson MC, Connor SJ, Milligan P, Flasse SP. Mapping malaria risk in Africa: what can satellite data contribute? Parasitol Today 1997 Aug;13(8):313-8. DOI PubMed

37. Gemperli A, Sogoba N, Fondjo E, Mabaso M, Bagayoko M, Briët OJ, Anderegg D, Liebe J, Smith T, Vounatsou P. Mapping malaria transmission in West and Central Africa. Trop Med Int Health 2006 Jul;11(7):1032-46. DOI PubMed

38. Beck LR, Lobitz BM, Wood BL. Remote sensing and human health: new sensors and new opportunities. Emerg Infect Dis 2000 May-Jun;6(3):217-27. DOl PubMed

39. Clements AC, Reid HL, Kelly GC, Hay SI. Further shrinking the malaria map: how can geospatial science help to achieve malaria elimination? Lancet Infect Dis 2013 Aug;13(8):70918. DOI PubMed

40. Correia VR, Carvalho MS, Sabroza PC, Vasconcelos CH. Remote sensing as a tool to survey endemic diseases in Brazil. Cad Saude Publica 2004 Jul-Aug;20(4):891-904. DOI PubMed

41. Agence spatiale canadienne (ASC). Satellites d'observation de la Terre. ASC, Canada. (Accédé janvier 2019). http://www. asc-csa.gc.ca/fra/satellites/default-ot.asp

42. National Aeronautics and Space Administration (NASA). AIRS Instrument Specs. NASA, United States of America (Accessed January 2019). https://airs.jpl.nasa.gov/mission_ and_instrument/instrument/specs

43. National Aeronautics and Space Administration (NASA). Precipitation measurement missions. United States of America (Accessed January 2019). https://pmm.nasa.gov/ precipitation-measurement-missions

44. National Aeronautics and Space Administration (NASA). Remote Sensors. NASA, United States of America (Accessed 
January 2019). https://earthdata.nasa.gov/user-resources/ remote-sensors

45. National Aeronautics and Space Administration (NASA). Suomi NPP Visible Infrared Imaging Radiometer Suite (VIIRS). NASA, United States of America (Accessed January 2019). https://ladsweb.modaps.eosdis.nasa.gov/missions-andmeasurements/viirs/

46. Remote Sensing Systems (RSS). Research-quality geophysical products from satellite microwave sensors. RSS, United States of America (Accessed January 2019). http://www. remss.com/

47. Satellite Imaging Corporation (SIC). Satellite Sensors. SIC, United States of America (Accessed January 2019). https:// www.satimagingcorp.com/satellite-sensors/

48. World Meteorological Organization (WMO). Observing Systems Capability Analysis and Review Tool (OSCAR). Switzerland: WMO; 2011-2019 (Accessed January 2019). https://www.wmo-sat.info/oscar/

49. Manore CA, Davis J, Christofferson RC, Wesson D, Hyman JM, Mores CN. Towards an early warning system for forecasting human west nile virus incidence. PLoS Curr 2014 Mar;6. DOl PubMed

50. Tran A, Sudre B, Paz S, Rossi M, Desbrosse A, Chevalier V, Semenza JC. Environmental predictors of West Nile fever risk in Europe. Int J Health Geogr 2014 Jul;13:26. DOI PubMed

51. Brown L, Medlock J, Murray V. Impact of drought on vector-borne diseases--how does one manage the risk? Public Health 2014 Jan;128(1):29-37. DOI PubMed

52. Rosà R, Marini G, Bolzoni L, Neteler M, Metz M, Delucchi L, Chadwick EA, Balbo L, Mosca A, Giacobini M, Bertolotti $L$, Rizzoli A. Early warning of West Nile virus mosquito vector: climate and land use models successfully explain phenology and abundance of Culex pipiens mosquitoes in north-western Italy. Parasit Vectors 2014 Jun;7:269. DOI PubMed

53. Gardner AM, Lampman RL, Muturi EJ. Land use patterns and the risk of West Nile virus transmission in central Illinois. Vector Borne Zoonotic Dis 2014 May; 14(5):338-45. DOI PubMed

54. Rees EE, Petukhova T, Mascarenhas M, Pelcat Y, Ogden $\mathrm{NH}$. Environmental and social determinants of population vulnerability to Zika virus emergence at the local scale. Parasit Vectors 2018 May;11(1):290. DOI PubMed

55. Barbour AG. Fall and rise of Lyme disease and other lxodes tick-borne infections in North America and Europe. Br Med Bull 1998;54(3):647-58. DOI PubMed

56. Kilpatrick AM, Dobson AD, Levi T, Salkeld DJ, Swei A, Ginsberg HS, Kjemtrup A, Padgett KA, Jensen PM, Fish D, Ogden NH, Diuk-Wasser MA. Lyme disease ecology in a changing world: consensus, uncertainty and critical gaps for improving control. Philos Trans R Soc Lond B Biol Sci 2017 Jun;372(1722):20160117. DOI PubMed

57. Gabriele-Rivet V, Koffi JK, Pelcat Y, Arsenault J, Cheng A, Lindsay LR, Lysyk TJ, Rochon K, Ogden NH. A Risk Model for the Lyme Disease Vector Ixodes scapularis (Acari: Ixodidae) in the Prairie Provinces of Canada. J Med Entomol 2017 Jul;54(4):862-8. DOI PubMed

58. Bouchard C, Beauchamp G, Leighton PA, Lindsay R, Bélanger D, Ogden NH. Does high biodiversity reduce the risk of Lyme disease invasion? Parasit Vectors $2013 \mathrm{Jul} ; 6: 195$. DOI PubMed

59. Cuber P, Andreassen Å, Vainio K, Asman M, Dudman S, Szilman P, Ottesen P, Anestad G, Cieśla-Nobis S, Solarz K. Risk of exposure to ticks (Ixodidae) and the prevalence of tick-borne encephalitis virus (TBEV) in ticks in Southern Poland. Ticks Tick Borne Dis 2015 Apr;6(3):356-63. DOI PubMed

60. Dantas-Torres F. Climate change, biodiversity, ticks and tick-borne diseases: the butterfly effect. Int J Parasitol Parasites Wildl 2015 Aug;4(3):452-61. DOI PubMed

61. Estrada-Peña A, de la Fuente J. The ecology of ticks and epidemiology of tick-borne viral diseases. Antiviral Res 2014 Aug;108:104-28. DOl PubMed

62. Werden L, Barker IK, Bowman J, Gonzales EK, Leighton PA, Lindsay LR, Jardine CM. Geography, deer, and host biodiversity shape the pattern of Lyme disease emergence in the Thousand Islands Archipelago of Ontario, Canada. PLoS One 2014 Jan;9(1):e85640. DOI PubMed

63. Ripoche M, Lindsay LR, Ludwig A, Ogden NH, Thivierge K, Leighton PA. Multi-Scale Clustering of Lyme Disease Risk at the Expanding Leading Edge of the Range of Ixodes scapularis in Canada. Int J Environ Res Public Health 2018 Mar;15(4):603. DOI PubMed

64. Batallán GP, Estallo EL, Flores FS, Sartor P, Contigiani MS, Almirón WR. St. Louis Encephalitis virus mosquito vectors dynamics in three different environments in relation to remotely sensed environmental conditions. Acta Trop 2015 Jun;146:53-9. DOI PubMed

65. Bowden SE, Magori K, Drake JM. Regional differences in the association between land cover and West Nile virus disease incidence in humans in the United States. Am J Trop Med Hyg 2011 Feb;84(2):234-8. DOI PubMed

66. Chuang TW, Henebry GM, Kimball JS, Vanroekel-Patton $\mathrm{DL}$, Hildreth MB, Wimberly MC. Satellite microwave remote sensing for environmental modeling of mosquito population dynamics. Remote Sens Environ 2012 Oct;125:147-56. DOI PubMed

67. DeGroote JP, Sugumaran R, Brend SM, Tucker BJ, Bartholomay LC. Landscape, demographic, entomological, and climatic associations with human disease incidence of West Nile virus in the state of lowa, USA. Int J Health Geogr 2008 May; 7:19. DOI PubMed

68. Liu $\mathrm{H}$, Weng $\mathrm{Q}$. Enhancing temporal resolution of satellite imagery for public health studies: A case study of West Nile Virus outbreak in Los Angeles in 2007. Remote Sens Environ 2012;117:57-71. DOI

69. vonHedemann N, Butterworth MK, Robbins P, Morin CW, Landau K. Visualizations of mosquito risk: A political ecology approach to understanding the territorialization of hazard control. Landscape Urban Plan. 2015;142:159-69. DOI 
70. Kotchi SO, Turgeon P, Michel P, Lavigne MP, Brazeau S. Assessing and Monitoring Microbiological Quality of Surface Waters Using Tele-Epidemiology. Glob Bioet 2011;24(14):65-70. DOI

71. United Nations Office for Outer Space Affairs (UNOOSA). Space Solutions for the World's Problems: How the United Nations family uses space technology for achieving development goals. Vienna (Austria): UNOOSA; 2005. http:// www.unoosa.org/oosa/oosadoc/data/documents/2005/ stspace/stspace200501_0.html

72. United Nations Office for Outer Space Affairs (UNOOSA). Charte Internationale Espace et Catastrophes Majeures. UNOOSA, Austria (Accédé janvier 2018). http://www.unspider.org/fr/applications-spatiales/mecanismes-durgences/ charte-internationale-espace-et-catastrophes-majeures

73. Asrar FM, Asrar S, Clark JB, Kendall DJ, Ngo-Anh TJ, Brazeau S, Hulsroj P, Williams RS. Help from above: outer space and the fight against Ebola. Lancet Infect Dis 2015 Aug;15(8):873-5. DOl PubMed

74. Yang C, Luo J, Hu C, Tian L, Li J, Wang K. An Observation Task Chain Representation Model for Disaster
Process-Oriented Remote Sensing Satellite Sensor Planning: A Flood Water Monitoring Application. Remote Sens 2018;10(3):375. DOI

75. Hamm NA, Soares Magalhães RJ, Clements AC. Earth Observation, Spatial Data Quality, and Neglected Tropical Diseases. PLoS Negl Trop Dis 2015 Dec;9(12):e0004164. DOI PubMed

76. Johnson DP, Wilson JS, Luber GC. Socioeconomic indicators of heat-related health risk supplemented with remotely sensed data. Int J Health Geogr 2009 Oct;8:57. DOI PubMed

77. Luber G, McGeehin M. Climate change and extreme heat events. Am J Prev Med 2008 Nov;35(5):429-35. DOI PubMed

78. Hazaymeh K, Hassan QK. Fusion of MODIS and landsat-8 surface temperature images: a new approach. PLoS One 2015 Mar;10(3):e0117755. DOI PubMed

79. Gouvernement du Canada. Innovation, Sciences et Développement économique Canada. Système de traitement et gestion des images d'observation de la Terre. ISDE. (Accédé décembre 2018). http://www.ic.gc.ca/eic/ site/101.nsf/fra/00029.html 